\title{
The Physical Society's Discussion on Magnetism.
}

THE discussion on magnetism organised by the Physical Society and held on May 23, at the Imperial College of Science and Technology, admirably illustrates the thesis that with advancing knowledge our concepts of a particular region of Nature, even though they may serve to correlate a wider range of phenomena, become less clear cut and more diffuse and difficult to follow.

When Sir Alfred Ewing formulated his magnetic models, our knowledge (in great measure due to his own happily conceived experiments) was confined mainly to the properties of the ferro-magnetic elements, and that knowledge could be subsumed under certain relatively simple concepts. Sir Alfred, in his opening address, reminded us that his own model, though not to be regarded as anything more than a piece of symbolism, could still assist in clearing our views concerning certain ferro-magnetic properties. He illustrated this position by instancing a criticism of the model made by Swinburne, who pointed out that its behaviour indicated the unlikely condition of a zero hysteresis loss in a revolving field strong enough to produce saturation. Though the fact has almost escaped notice, experiments by Baily on the hysteresis loss in a revolving field have shown that the loss increases with increasing field strength to a maximum and then does fall almost to zero when the field is strong enough to produce saturation.

About the beginning of this century, Curie's investigations, developing the fundamental observations of Faraday, had resulted in the well-known generalisations that the mass-susceptibility of a diamagnetic was independent of the temperature, and of a paramagnetic varied inversely as the absolute temperature. The concept of the magneton was shadowed forth so far back as the eighteen-seventies by Weber, in a model identical with that of simple electron theory, save that the roles of the electricities are reversed. Weiss gave the name to a fundamental unit of magnetic moment obtained from a study of the masssusceptibility per gram-atom, and a knowledge of Avogadro's number, the number of atoms in the gram-atom. Such atomic magnetic moments were alwavs found to be integral multiples of a fundamental quantity, the Weiss magneton, $18.54 \times 10^{-22}$.

It is easy to calculate, by the classical laws, the magnetic moment of the magnet competent to produce the field due to an electron revolving in a circular orbit about a central nucleus. Writing down the angular momentum of the circulating electron, taking the ratio of the magnetic moment to the angular momentum, and in terms of the quantum theory assuming the angular momentum to be an integral multiple of $h / 2 \pi$, we easily find in the simplest case that the magnetic moment of the Bohr magneton is $h e / 4 \pi m$, which, by substitution of known values, gives the magnetic moment of the Bohr magneton as almost exactly five times that of the Weiss magneton. The properties of a ' ring' electron, in which the revolving charge, instead of being concentrated at a point, is uniformly distributed over a thin anchor ring, have from time to time been discussed, and it is interesting to note that some of Schrodinger's recent results are strictly analogous to those obtained for this ring type of magneton.

Langevin's classical paper of 1905 gave a basis for Curie's experimental generalisations concerning the behaviour with temperature of dia- and paramagnetic bodies. Again an appeal is made to classical methods. The electron orbit behaves as a magnet and possesses a magnetic moment. It is possible that, for a given molecule, these orbital moments give no resultant magnetic moment. The application of a field which rises from zero to some final value, $H$, will cause a change in the magnetic moment which can be calcu. lated on the assumption that an electromotive force is induced in the orbit, measured by the rate of change of the flux through it. It is not difficult to show that this change is negative. In a paramagnetic gas we have to deal with molecules which have a resultant magnetic moment, and, assuming an initial random distribution of the axes of these magnets, it may be shown that the application of a magnetic field results in the development of a magnetic moment giving a mass-susceptibility varying inversely as the absolute temperature.

So far the effect of any mutual molecular interaction has been neglected, but if, following Weiss, we assume the existence of a molecular field proportional always to the intensity of magnetisation, $I$, so that the total field is $(H+N I)$, we then obtain for the mass-susceptibility the modified Curie law, which makes the susceptibility inversely proportional, not to $T$, but to $(T-\theta)$. But considerations of the Zeeman effect show that Langevin's original treatment of paramagnetism requires serious modification, inasmuch as we find that only certain discrete values can be assumed by the resolutes, in the field-direction, of the atomic magnetic moments, and in order to resolve the difficulties of the situation it is necessary to endow the electron with an intrinsic spin and a magnetic moment.

These elementary considerations and many, more points of importance in modern theory are developed in the contributions made to the discussion by Dr. E. C. Stoner and by Prof. H. S. Allen.

Prof. C. G. Darwin discussed the polarisation of the electron. The quantisation of the electronic orbit leaves unexplained certain finer phenomena which are covered by giving the electron itself an intrinsic spin, and now with the development of wave mechanics comes the notion that the electron can be considered as a polarised wave. Prof. Darwin considered in some detail the possibility of observing directly the magnetic moment of a free electron, as distinguished from the indirect verification observed by loading the electron with the core of a silver atom and subjecting the stream of atorns to the action of a non-uniform magnetic field as in the experiments of Gerlach and Stern.

Bohr has suggested that any experiment of this type carried out on streams of free electrons would necessarily give negative results, as the electrons would have uncertainties of position and speed which would blot out any systematic effect which we might hope to obtain. Prof. Darwin disagrees with this conclusion, based as it is on the assumption that the behaviour of the polarised electrons is observed by one magnetometer, whereas a survey of the magnetic field by several magnetometers may enable us to overcome the uncertainty.

Mr. W. Sucksmith discussed some difficult experiments on the measurement, for ferro- and paramagnetics, of the gyromagnetic ratio-..the ratio of the magnetic moment of the magneton to its angular momentum-a knowledge of which leads to an estimate of the Landé splitting factor $g$. For the Dy ${ }^{+++}$ ion in $\mathrm{Dy}_{2} \mathrm{O}_{3}$ he finds for $g$ the value $1 \cdot 28 \pm 7$ per cent, the theoretical value being $4 / 3$.

Prof. W. Peddie's contribution dealt with the interrelations of magnetisation and temperature in crystals from the point of view of kinetic theory. He showed that a good general account of the phenomena can be given by a reduced equation of state of the second degree. Dr. W. L. Webster gave an account of magnetostriction and change of resistance in single crystals of iron and nickel, showing that there are two

No. 3162, VoL. 125] 
stages in the process of magnetisation which involve different distortions of the crystal.

Dr. L. F. Bates described some experiments on the temperature-variation of the specific heat of manganese arsenide, which has a critical temperature in the neighbourhood of $45^{\circ} \mathrm{C}$. The hypothesis of an internal field, put forward by Weiss, leads to a definite shape for the specific heat-temperature curve, and Dr. Bates finds that while the general form of the curve is similar to that given by the Weiss theory, the variation of specific heat with temperature is more nearly proportional to $d I_{0} / d T^{\prime}$ than to $d I_{0}{ }^{2} / d T$.

Mr. F. C. Powell dealt with the change in size of a ferro-magnetic at the Curie point. A rough estimate of its magnitude has already been made for iron on Heisenberg's theory, but the experimental values had been misquoted. The present paper corrects this and also revises the theoretical estimate in the light of Heisenberg's morified theory. The numerical values for iron and nickel are calculated and are found to be of the order demanded by the theory.

Prof. Weiss and M. Forrel gave the results of an experimental study of the saturation data for a number of ferro-cobalts and nickel-cobalts and deduced therefrom the atomic moments of iron, nickel, and cobalt.

The paper by Prof. W. Gerlach is an experimental study of the relation between certain electrical and ferro-magnetic properties. Nickel is the substance under investigation and its change of resistance with temperature has been studied over a range which includes the Curie point. 'The curves showing the variation with temperature of the temperaturecoefficient of the resistance are in very close agreement with the curves obtained by Weiss for the variation of specific heat with temperature. Prof. Gerlach further discusses the resistance-change of nickel in a longitudinal field and describes a new thermo-magnetic effect. If a ferro-magnetic body is placed in a homogeneous magnetic field, and a temperature difference is established between its ends, the lines of force of the field coinciding in direction with the line of temperature fall, an e.m.f. appears between the ends of the body. Prof. Gerlach describes preliminary experiments on the variation of this e.m.f. with variation of the temperature at the ends of the specimen.

It need scarcely be said that Dr. P. Kapitza's account of his remarkable experiments in strong fields was full of interest, and it is not too much to assert that a technique which has placed in the hands of experimenters a method for developing fields of 300 kilo-gauss in a volume of 3 cubic centimetres, even though the duration of the field be a hundredth of a second, represents one of the most important advances in experimental work that the last few years has afforded. The magnification of the ordinary effects observed reaches such large values that the short space of time in which the effects are observable introduces no unsurmountable difficulties. Thus the splitting of the lines in the Zeeman effect is so large that it may be observed by means of the ordinary prism spectrograph, and changes of electrical resistance which in ordinary fields require very special methods for their estimation are, in these large fields, increases of 20 or 30 per cent.

The whole discussion was thoroughly enjoyable. If it tended to show that modern magnetics, in some of its aspects, is still in a pre-Newtonian stage, where it is dominated by

"Cycle on epicycle, orb on orb,

With centric and eccentric scribbled o'er,"

it has cleared up some difficulties, focused attention on others, and has certainly helped to systematise the ideas of many of those who were present.

\section{ALIAN FERGuson.}

\section{Utilisation of Potatoes.}

A PRACTICAL account showing how potatoes can

be used most profitably at present prices is given in the Scottish Journal of Agriculture, vol. 13, p. 30 . Both potatoes and oats have a high feeding value, and on farms carrying stock it is being found more economical to use them for food than to sell them. The quantities that can be safely used, their relative food values and costs, are compared with those of other commonly used food stuffs, data from actual feeding experiments being quoted in support of the recommendations.

Cattle can utilise potatoes raw, but for pigs they are better cooked; green or sprouted tubers should be avoided as they are liable to be harmful. Since the protein and mineral content of potatoes is low, a supplementary ration such as separated $\mathrm{milk}$ and a mineral mixture is necessary for young animals or dairy cows. On a ration containing a large proportion of oats and boiled potatoes so supplemented, young pigs gave as good gains, at lower cost., as when raised on other commonly used food stuffs.

The question as to the best use to make of surplus potatoes is also discussed. One of the chief difliculties lies in the fact that the surplus is a casual one, depending on seasonal conditions, although importation of foreign potatoes and overplanting contribute to the problem. The possible uses, other than human consumption, to which potatoes can be put are various. Feeding to stock, either whole or as silage, is probably the simplest way of dealing with the surplus, but silage-making needs further investigation and development in England. Alcohol, starch, acetic acid, or dried potatoes are successfully produced from potatoes on the continent, but so far little has been done in this direction in Great Britain. The fluctuating supplies of raw material present a special difficulty in establishing such industries.

Increasing the demand by selection for cooking quality rather than yield, increased export of seed, improved grading and better marketing, especially in early summer when imports are greatest, are further means by which the surplus may be reduced. In fact, better organisation, together with a diversion of the surplus into channels other than for human consumption, is necessary to make potato-growing in Great Britain remunerative and a trustworthy source of profit.

\section{New Short-Circuit Testing Plant.}

WE are glad to notice that the number of commercial testing laboratories in Great Britain is rapidly increasing. As a rule these laboratories are not co-operative but are set up by individual companies. In the Electrical Review for May 30 there is an interesting account of a short-circuit testing plant which has been erected by Messrs. A. Reyrolle and Co., of Hebburn-on-Tyne.

The plant has the short-circuit capacity of $1,500,000$ kilovolt amperes and is the largest plant of this kind in Great Britain. It is situated at Reyrolles' New Town Works between Newcastle and South Shields. As large devices have to be tested, in some cases until they break down and are destroyed, and as huge amounts of electric energy are suddenly converted into heat, great precautions have to be taken to prevent fire. Cylinders filled with carbon dioxide are stored in the transformer room. The gas can be released by a switch in the control house or locally by hand and the machine and terminal pit are at once flooded with the gas.

No. 3162 , VoL. 125] 